\title{
BOUNDING THE OPEN $k$-MONOPOLY NUMBER OF STRONG PRODUCT GRAPHS
}

\author{
Dorota KUZIAK \\ Departamento de Estadística e Investigación Operativa \\ EPS, Universidad de Cádiz \\ Av. Ramón Puyol s/n, 11202 Algeciras, Spain \\ e-mail: dorota.kuziak@uca.es \\ IZTOK PETERIN \\ University of Maribor, FEECS \\ Koroška 46, 2000 Maribor, Slovenia \\ and \\ IMFM, Jadranska 19, 1000 Ljubljana, Slovenia \\ e-mail: iztok.peterin@um.si \\ AND \\ ISMAEL G. YeRO \\ Departamento de Matemáticas, EPS, Universidad de Cádiz \\ Av. Ramón Puyol s/n, 11202 Algeciras, Spain \\ e-mail: ismael.gonzalez@uca.es
}

\begin{abstract}
Let $G=(V, E)$ be a simple graph without isolated vertices and minimum degree $\delta$, and let $k \in\{1-\lceil\delta / 2\rceil, \ldots,\lfloor\delta / 2\rfloor\}$ be an integer. Given a set $M \subset V$, a vertex $v$ of $G$ is said to be $k$-controlled by $M$ if $\delta_{M}(v) \geq \frac{\delta_{G}(v)}{2}+k$, where $\delta_{M}(v)$ represents the number of neighbors of $v$ in $M$ and $\delta_{G}^{2}(v)$ the degree of $v$ in $G$. A set $M$ is called an open $k$-monopoly if every vertex $v$ of $G$ is $k$-controlled by $M$. The minimum cardinality of any open $k$-monopoly is the open $k$-monopoly number of $G$. In this article we study the open $k$ monopoly number of strong product graphs. We present general lower and upper bounds for the open $k$-monopoly number of strong product graphs. Moreover, we study in addition the open 0-monopolies of several specific families of strong product graphs.
\end{abstract}

Keywords: open monopolies, strong product graphs, alliances, domination.

2010 Mathematics Subject Classification: 05C69, 05C76. 


\section{REFERENCES}

[1] D.W. Bange, A.E. Barkauskas and P.J. Slater, Efficient dominating sets in graphs, in: Applications of Discrete Math., R.D. Ringeisen and F.S. Roberts (Ed(s)), (SIAM, Philadelphia, 1988) 189-199.

[2] N. Biggs, Perfect codes in graphs, J. Combin. Theory Ser. B 15 (1973) 289-296. doi:10.1016/0095-8956(73)90042-7

[3] C. Dwork, D. Peleg, N. Pippenger and E. Upfal, Fault tolerance in networks of bounded degree, SIAM J. Comput. 17 (1988) 975-988. doi:10.1137/0217061

[4] H. Fernau, J.A. Rodríguez-Velázquez and J.M. Sigarreta, Global powerful r-alliances and total k-domination in graphs, Util. Math. 98 (2015) 127-147.

[5] P. Flocchini, R. Královič, A. Roncato, P. Ružička and N. Santoro, On time versus size for monotone dynamic monopolies in regular topologies, J. Discrete Algorithms 1 (2003) 129-150. doi:10.1016/S1570-8667(03)00022-4

[6] H. García-Molina and D. Barbara, How to assign votes in a distributed system, J. ACM 32 (1985) 841-860. doi: $10.1145 / 4221.4223$

[7] R. Hammack, W. Imrich and S. Klavžar, Handbook of Product Graphs, Second Edition (CRC Press, Boca Raton, FL, 2011).

[8] K. Khoshkhah, M. Nemati, H. Soltani and M. Zaker, A study of monopolies in graphs, Graphs Combin. 29 (2013) 1417-1427. doi:10.1007/s00373-012-1214-7

[9] P. Kristiansen, S.M. Hedetniemi and S.T. Hedetniemi, Alliances in graphs, J. Combin. Math. Combin. Comput. 48 (2004) 157-177.

[10] D. Kuziak, I. Peterin and I.G. Yero, Computing the $(k-)$ monopoly number of direct product of graphs, Filomat 29 (2015) 1163-1171. doi:10.2298/FIL1505163K

[11] D. Kuziak, I. Peterin and I.G. Yero, On the monopolies of lexicographic product graphs: bounds and closed formulaes, Bull. Math. Soc. Sci. Math. Roumanie N.S. 59 (2016) 355-366.

[12] D. Kuziak, I. Peterin and I.G. Yero, Open $k$-monopolies in graphs: complexity and related concepts, Discrete Math. Theor. Comput. Sci. 18 (3) (2016).

[13] N. Linial, D. Peleg, Yu. Rabinovich and M. Saks, Sphere packing and local majorities in graphs, in: Proc. 2nd Israel Symposium on Theory and Computing Systems (Natanya, Israel, 1993) 141-149. doi:10.1109/ISTCS.1993.253475

[14] A. Mishra and S.B. Rao, Minimum monopoly in regular and tree graphs, Discrete Math. 306 (2006) 1586-1594. doi:10.1016/j.disc.2005.06.036 
[15] D. Peleg, Local majorities, coalitions and monopolies in graphs: a review, Theoret. Comput. Sci. 282 (2002) 231-257. doi:10.1016/S0304-3975(01)00055-X

[16] I. Peterin, The complexity of open $k$-monopolies in graphs for negative $k$, manuscript (2016).

[17] G.F. Sullivan, Complexity of System-Level Fault Diagnosis and Diagnosability, Ph.D. Thesis (Yale University, New Haven, CT, USA, 1986).

[18] I.G. Yero and J.A. Rodríguez-Velázquez, A survey on alliances in graphs: defensive alliances, Util. Math. (2016), to appear.

[19] M. Zaker, On dynamic monopolies of graphs with general thresholds, Discrete Math. 312 (2012) 1136-1143.

doi:10.1016/j.disc.2011.11.038

Received 26 November 2015

Revised 15 December 2016

Accepted 19 December 2016 